\title{
REVISTA
}

Revista Educación

ISSN: 0379-7082

ISSN: 2215-2644

revedu@gmail.com

Universidad de Costa Rica

Costa Rica

\section{Inclusión y justicia social en México. ¿Qué hacer desde la educación?}

Pérez Archundia, Eduardo; Millán Valenzuela, Henio

Inclusión y justicia social en México. ¿Qué hacer desde la educación?

Revista Educación, vol. 43, núm. 2, 2019

Universidad de Costa Rica, Costa Rica

Disponible en: http://www.redalyc.org/articulo.oa?id=44058158043

DOI: https://doi.org/10.15517/revedu.v43i2.34047

Esta obra está bajo una Licencia Creative Commons Atribución-NoComercial-SinDerivar 3.0 Internacional. 


\title{
Inclusión y justicia social en México. ¿Qué hacer desde la educación?
}

\author{
Inclusion and Social Justice in Mexico. What can be done from an Educational Perspective?
}

Eduardo Pérez Archundia

DOI: https://doi.org/10.15517/revedu.v43i2.34047

Instituto Superior de Ciencias de la Educación del Estado de Redalyc: http://www.redalyc.org/articulo.oa?id=44058158043

México, México

eperarc@hotmail.com

(D) http://orcid.org/0000-0003-2685-7347

Henio Millán Valenzuela

El Colegio Mexiquense, AC, México

hmillan@cmq.edu.mx

(iD http://orcid.org/0000-0003-0115-0636

Recepción: 21 Enero 2019

Aprobación: 26 Junio 2019

\section{RESUMEN:}

México, en el marco de una crisis social, atraviesa un proceso de alternancia electoral que para muchos representa el inicio de una nueva forma de hacer política, lo cual trae consigo la esperanza de acabar con la pobreza, la inseguridad y el deterioro educativo. A partir de este escenario, el presente ensayo contiene una discusión sobre las condiciones de premodernidad que prevalecen en México y obstaculizan el desarrollo económico, educativo y de seguridad social, con lo cual se pretende aportar elementos para visualizar la complejidad de factores involucrados, aunque manifestados en diferentes maneras, en el deterioro social. El documento señala la forma en que están imbricadas la pobreza, delincuencia y educación, al tiempo que apunta la necesidad de contener el abandono escolar en el nivel medio superior, lo cual está vinculado con dificultades para incorporarse al mercado laboral y su consecuente relación con la pobreza, de igual forma, el abandono escolar se constituye en un factor más que estimula la comisión de delitos. Al final del manuscrito se plantea la educación como una de las vías para abatir la pobreza y la delincuencia, pero no la educación que actualmente se imparte, primero tendrían que resolverse los problemas de calidad académica, la deserción en nivel medio superior y superior, así como la cobertura en estos niveles educativos. Esto demanda una educación diferenciada.

Palabras Clave: Pobreza, Delincuencia, Exclusión.

\section{Abstract:}

Mexico is currently undergoing a social crisis. On the one hand, it experiencing alternating elections which many consider to be the advent of a new form of politics which also brings with it the hope of ending poverty, insecurity and deterioration of the educational system. Based on this scenario, this article discusses premodern conditions that are prevalent in Mexico and which have obstructed the development of the country's economy, educational and social security sectors. It also highlights the complexity of the different types of factors that involved in social deterioration. The article questions how poverty, crime and education are superimposed and emphasizes the need to contain high school drop-out rates which are tied to difficulties in entering the job market and its consequential relationship to poverty. Dropping out of school also encourages youngsters to commit crimes. Education is perceived as one of the solutions to fight crime and poverty - however, not education as it currently stands. Academic problems would first have to be resolved, such as preventing school dropouts in both High School and College as well as improving coverage, which requires a differentiated type of education.

KEYWORDS: Poverty, Delinquency, Exclusion.

\section{INTRODUCCIÓN}

Hay quienes manifiestan que México vivió una fiesta democrática el pasado $1^{\circ}$ de julio de 2018. Los resultados de las elecciones para presidente, diputados, senadores y otros cargos locales, dieron como vencedor indiscutible al partido MORENA (Movimiento de Regeneración Nacional), lo que trajo consigo un nuevo 
proceso de alternancia en el poder ejecutivo federal y la reconfiguración de fuerzas políticas en todos los órdenes de gobierno. En la plataforma política del candidato ganador a la Presidencia de la República Mexicana, se encontraba el combate a la pobreza, la austeridad fiscal, la derogación de la reforma educativa promulgada por el presidente saliente, la amnistía para cierto tipo de delitos y una nueva estrategia de combate al narcotráfico, entre otras cosas, para tener como resultado final una sociedad en paz y con mayor bienestar.

Así, es pertinente analizar objetivamente el sentido de las acciones anunciadas por el presidente electo, dejando de lado filiaciones partidistas, la pertenencia o identificación con ciertos sectores de la población, así como la posición que un actor particular pueda tener dentro de este sistema social que ya hace muchos años presenta una fuerte crisis. Pero, sobre todo, el análisis objetivo de esta realidad debe favorecer la comprensión de la complejidad de los problemas en cuestión y de las alternativas de solución las cuales tienen los tomadores de decisiones, no solo como buenas intenciones sino como acciones planificadas con objetivos claros y conexos que redunden en bienestar y paz social. ¿Es posible combatir la pobreza sin mejorar la educación? ¿La mejor estrategia de combate al narcotráfico es reducir la pobreza? ¿La comisión de delitos tiene alguna relación con la pobreza y la educación? Estas son algunas interrogantes que señalan la forma en que pueden estar relacionadas tres de las prioridades nacionales: reducir la violencia social, reducir la pobreza y mejorar la educación.

Como a continuación se diserta, la política educativa no puede operar aislada de la economía y la seguridad pública. En tiempos electorales surgen voces clamando el origen de la crisis social en la pobreza, puesto que los asaltos, robos, extorsiones, etcétera, son cometidos por gente que se encuentra en condición de marginación. A esta cadena de eventos se suma uno más, la falta de oportunidades de educación. Por lo tanto, pareciera que la solución a la pobreza y la delincuencia se encuentra en la educación: una sociedad educada no será pobre ni se involucrará en actos delictivos. Si bien estos dichos tienen razón desde el sentido común, representan una respuesta simplista a dos de los problemas sociales prioritarios; la ecuación no se reduce a: mayor educación igual a menos pobreza y delincuencia.

El diseño de políticas públicas en materia educativa deberá considerar que para tener mayor calidad educativa también deben implementarse políticas sociales que, entre otras cosas, disminuyan la pobreza. Ahora bien, en cuanto a la prevención del delito, también debe hacerse un análisis más complejo pues, aunque la pobreza guarde relación cercana con la delincuencia, no es el único factor que debe atenderse para alcanzar condiciones de mayor estabilidad y paz social.

Desde el ámbito académico no puede reducirse el análisis, las categorías en cuestión son interdependientes, por lo cual, el objetivo de este ensayo es analizar la imbricación que existe entre educación, pobreza y delincuencia en el marco de la sociedad mexicana. Cabe señalar que no se pretende hacer recomendaciones para el diseño u operación de políticas públicas, solo enriquecer el debate en torno a estos tópicos.

Para ilustrar en lo general el escenario que vive México en torno a las tres categorías antes mencionadas, se presentan algunos indicadores por entidad federativa. El número de delitos del fuero común (Secretariado Ejecutivo del Sistema Nacional de Seguridad Pública, 2017), entre los cuales se encuentran: robos, lesiones, homicidios, delitos patrimoniales, secuestro, violación y otros delitos como amenazas, estupro y sexuales. En cuanto a la pobreza se retoma la medición por estado del número de personas en situación de pobreza (Consejo Nacional de Evaluación de la Política de Desarrollo Social, 2016), mientras que en educación se consideró la cobertura en todos los niveles educativos, abandono y eficiencia en educación media superior y superior, promedio de escolaridad y analfabetismo (Sistema Nacional de Información y Estadística Educativa, 2017).

\section{CiFRAS DE DELINCUENCIA, POBREZA Y EDUCACIÓN}

El expresidente de México, Enrique Peña Nieto, implementó una reforma educativa de la cual se deriva el Nuevo Modelo Educativo, presentado en 2017 por la Secretaría de Educación Pública, con el cual se pretende 
ofrecer una educación de calidad que contribuya al desarrollo pleno del potencial de todas las niñas, niños y jóvenes, para ser personas exitosas en el siglo XXI (Secretaría de Educación Pública, 2017). Entre sus planteamientos se puede encontrar la implementación de estrategias que favorezcan la inclusión y equidad dentro del sistema educativo, con lo que se pretende, a su vez, que todos los actores sociales sean integrados a la educación formal en condiciones de equidad, no solo en el sistema educativo sino también en el sistema social. Sin embargo, el primer elemento a cuestionar es el alcance que el Nuevo Modelo Educativo pueda tener, tomando en cuenta que está enfocado principalmente hacia la inclusión en educación básica, la educación superior no está considerada y el bachillerato se encuentra presente de forma adyacente.

Lo anterior cobra relevancia debido a que, en primer término, los problemas de cobertura educativa no se encuentran en el nivel básico, $96.4 \%$ a nivel nacional, sino en bachillerato, $76.6 \%$, y nivel superior, $32.1 \%$ (ver Tabla 1). Este último dato disminuye aún más si se considera el posgrado, 28.7\% (Sistema Nacional de Información y Estadística Educativa, 2017). Aunado a esto, debe considerarse que el mayor porcentaje de abandono escolar se presenta en el bachillerato, 12.8\%, seguido por la educación superior con $6.8 \%$. En consecuencia, el grado promedio de escolaridad a nivel nacional es de 9.3, lo que ubica el bachillerato por encima de la norma, vulnerando así la inserción de las y los jóvenes en el sistema social, como señala Huang (2016): los estudiantes que abandonan sus estudios en nivel medio superior limitan sus oportunidades sociales debido a que no cuentan con las calificaciones necesarias para su integración en el mercado laboral y corren un alto riesgo de exclusión social y pobreza, además, tienen mayores dificultades para participar en la vida cívica y democrática. Por lo tanto, en educación media el énfasis debe ser evitar la deserción. 
TABLA 1

Indicadores educativos, pobreza y delitos del fuero común por estado

\begin{tabular}{|c|c|c|c|c|c|c|c|c|c|c|c|}
\hline & \multirow{2}{*}{ Analfabetismo } & \multirow{2}{*}{\begin{tabular}{|l|}
$\begin{array}{c}\text { Educación } \\
\text { Básica \% }\end{array}$ \\
Cobertura \\
(3 a 14 \\
años de \\
edad)
\end{tabular}} & \multicolumn{3}{|c|}{ Educación Media Superior \% } & \multicolumn{3}{|c|}{ Educación Superior \% } & \multirow[b]{2}{*}{$\begin{array}{l}\text { Grado } \\
\text { Promedio } \\
\text { de } \\
\text { Escolaridad }\end{array}$} & \multirow[b]{2}{*}{ Pobreza } & \multirow[b]{2}{*}{$\begin{array}{l}\text { Delitos del } \\
\text { fuero } \\
\text { común }\end{array}$} \\
\hline & & & $\begin{array}{l}\text { Abandono } \\
\text { escolar }\end{array}$ & \begin{tabular}{|l|} 
Eficiencia \\
Terminal
\end{tabular} & \begin{tabular}{|l|} 
Cobertura \\
(15 a 17 \\
años de \\
edad)
\end{tabular} & $\begin{array}{l}\text { Abandono } \\
\text { escolar }\end{array}$ & \begin{tabular}{|} 
Cobertura \\
(Incluye \\
Posgrado) \\
(18a 23 \\
años de \\
edad)
\end{tabular} & \begin{tabular}{|l|} 
Cobertura \\
(No \\
Incluye \\
Posgrado) \\
(18 a 22 \\
años de \\
edad)
\end{tabular} & & & \\
\hline Nacional & 4.7 & 96.4 & 12.8 & 66.6 & 76.6 & 6.8 & 28.7 & 32.1 & 9.3 & $53,418,151$ & $1,807,846$ \\
\hline Aguascalientes & 1.1 & 94.0 & 13.3 & 68.8 & 77.6 & 6.2 & 35.1 & 40.4 & 9.9 & 369,652 & 33,548 \\
\hline $\begin{array}{l}\text { Baja } \\
\text { California }\end{array}$ & 1.6 & 92.0 & 16.3 & 60.8 & 84.9 & 3.7 & 30.4 & 34.3 & 9.8 & 789,109 & 101,466 \\
\hline $\begin{array}{l}\text { Baja } \\
\text { California Sur }\end{array}$ & 1.8 & 85.7 & 13.7 & 66.1 & 79.7 & 14.7 & 27.7 & 32.0 & 10.0 & 175,642 & 24,174 \\
\hline Campeche & 5.9 & 94.0 & 16.6 & 61.5 & 73.7 & 11.4 & 27.6 & 32.3 & 9.3 & 405,035 & 1,924 \\
\hline Coahuila & 1.7 & 96.7 & 15.0 & 64.9 & 72.9 & 8.5 & 30.9 & 34.8 & 10.0 & 745,897 & 56,311 \\
\hline Colima & 3.1 & 91.3 & 13.3 & 67.8 & 76.7 & 10.2 & 28.3 & 32.6 & 9.6 & 248,691 & 24,424 \\
\hline Chiapas & 13.9 & 103.7 & 13.0 & 64.5 & 69.3 & 3.1 & 13.0 & 14.5 & 7.4 & $4,113,950$ & 25,364 \\
\hline Chihuahua & 1.9 & 88.8 & 14.8 & 63.2 & 74.3 & 5.7 & 33.2 & 37.7 & 9.4 & $1,149,961$ & 68,819 \\
\hline $\begin{array}{l}\text { Ciudad de } \\
\text { México }\end{array}$ & 1.2 & 106.8 & 16.3 & 52.6 & 119.7 & 3.6 & 66.6 & 70.0 & 11.0 & $2,434,424$ & 204,078 \\
\hline Durango & 2.7 & 97.7 & 17.3 & 60.5 & 76.6 & 5.2 & 25.3 & 27.9 & 9.3 & 643,299 & 34,851 \\
\hline Guanajuato & 5.5 & 97.9 & 15.5 & 65.5 & 68.2 & 2.6 & 21.1 & 23.2 & 8.4 & $2,489,715$ & 117,857 \\
\hline Guerrero & 11.4 & 99.8 & 13.1 & 68.7 & 65.6 & 4.7 & 16.4 & 19.2 & 7.9 & $2,314,720$ & 32,799 \\
\hline Hidalgo & 7.0 & 99.3 & 13.7 & 64.2 & 83.4 & 5.0 & 31.3 & 35.4 & 8.9 & $1,478,836$ & 43,963 \\
\hline Jalisco & 3.2 & 95.4 & 2.7 & 85.7 & 73.2 & 2.2 & 27.5 & 30.9 & 9.4 & $2,560,592$ & 117,554 \\
\hline México & 2.7 & 92.3 & 13.7 & 65.1 & 70.4 & 9.3 & 24.1 & 27.2 & 9.6 & $8,230,200$ & 291,003 \\
\hline Michoacán & 6.8 & 96.1 & 12.8 & 65.8 & 67.3 & 9.9 & 20.8 & 23.8 & 8.0 & $2,565,862$ & 41,836 \\
\hline Morelos & 4.1 & 93.1 & 17.0 & 61.9 & 83.5 & 4.0 & 29.6 & 32.6 & 9.5 & 965,852 & 44,329 \\
\hline Nayarit & 4.3 & 87.8 & 18.1 & 54.2 & 80.9 & 5.0 & 26.0 & 30.1 & 9.3 & 470,130 & 3,220 \\
\hline Nuevo León & 1.4 & 98.7 & 9.0 & 78.0 & 71.2 & 2.2 & 37.9 & 42.1 & 10.2 & 737,765 & 83,974 \\
\hline Oaxaca & 11.7 & 101.4 & 12.7 & 67.1 & 64.0 & 8.9 & 15.4 & 17.7 & 7.6 & $2,847,341$ & 31,585 \\
\hline Puebla & 7.2 & 101.1 & 10.5 & 76.2 & 78.1 & 9.5 & 32.8 & 36.3 & 8.6 & $3,728,202$ & 53,800 \\
\hline Querétaro & 3.0 & 101.2 & 10.7 & 70.1 & 79.3 & 0.4 & 32.0 & 35.5 & 9.6 & 635,680 & 53,379 \\
\hline Quintana Roo & 3.4 & 89.6 & 12.0 & 69.8 & 72.7 & 13.9 & 20.3 & 23.8 & 9.9 & 471,040 & 26,518 \\
\hline $\begin{array}{l}\text { San Luis } \\
\text { Potosí }\end{array}$ & 5.2 & 97.6 & 13.1 & 67.8 & 67.3 & 10.0 & 24.9 & 28.4 & 8.9 & $1,267,728$ & 35,179 \\
\hline Sinaloa & 3.8 & 93.8 & 11.3 & 69.9 & 80.5 & 8.7 & 38.4 & 44.4 & 9.8 & 929,683 & 22,931 \\
\hline Sonora & 1.8 & 87.4 & 13.5 & 66.1 & 80.8 & 13.2 & 34.7 & 39.9 & 10.1 & 831,427 & 25,969 \\
\hline Tabasco & 5.0 & 104.1 & 11.1 & 68.6 & 83.6 & 11.3 & 27.6 & 31.9 & 9.4 & $1,228,092$ & 60,395 \\
\hline Tamaulipas & 2.4 & 90.1 & 13.4 & 65.2 & 75.8 & 8.9 & 31.0 & 35.6 & 9.6 & $1,156,220$ & 47,163 \\
\hline Tlaxcala & 3.1 & 94.4 & 13.6 & 64.4 & 75.2 & 3.9 & 23.8 & 27.2 & 9.4 & 701,757 & 6,964 \\
\hline Veracruz & 8.0 & 93.2 & 10.4 & 72.8 & 78.2 & 12.3 & 21.7 & 24.7 & 8.3 & $5,049,511$ & 49,205 \\
\hline Yucatán & 6.6 & 95.6 & 13.9 & 62.2 & 81.8 & 8.2 & 31.5 & 35.6 & 9.0 & 901,864 & 24,390 \\
\hline Zacatecas & 2.9 & 99.6 & 13.6 & 64.0 & 75.7 & 12.0 & 27.9 & 31.6 & 8.7 & 780,274 & 18,874 \\
\hline
\end{tabular}

Fuente: Elaboración propia con datos de Consejo Nacional de Evaluación de la Política de Desarrollo Social (2016), Secretariado Ejecutivo del Sistema Nacional de Seguridad Pública (2017) y Sistema Nacional de Información y Estadística Educativa (2017). Nota: Los valores pico de cada indicador se encuentran sombreados.

El problema de acceso a la educación superior no surge repentinamente en el nivel superior, desde el bachillerato se van cerrando las oportunidades. En México, desde el año 2012, la educación media superior se volvió obligatoria por decreto, pero no ha funcionado así en la práctica. Los habitantes en situación de pobreza son incluidos en los niveles elementales de formación escolar sin que exista un diseño institucional que cree posibilidades reales de acceso hasta el nivel superior.

Estos datos llevan a pensar que el esfuerzo de las autoridades educativas en materia de inclusión, plasmado en el Nuevo Modelo Educativo, consiste en incrementar la cobertura y disminuir el abandono escolar en educación básica, para lo cual se expanden los servicios educativos en zonas marginadas y en comunidades rurales en las que aún no cuentan con una escuela, pero solo a nivel básico. Pese a esos esfuerzos, habría que analizar más allá de la cobertura y observar también la calidad de los servicios educativos ofrecidos en zonas urbanas y marginadas. De acuerdo con Li (2016), para erradicar la desigualdad regional de educación, que a su vez se relaciona con desigualdad social, es necesario distribuir simétrica y funcionalmente los recursos educativos, tomando en cuenta los procesos de migración rural - urbana que suelen alterar las cifras oficiales de residentes por localidad, lo cual ocasiona inequidad en el acceso a la educación. Así se podrían atender dos condiciones: a) combatir el analfabetismo mediante una cobertura total de educación básica, 
para contrarrestar un factor que vulnera a la población e incentiva las posibilidades de caer en situación de pobreza, tal como afirman la Comisión Económica para América Latina y el Caribe (2009) y Di Lorenzo y Rosales (2013); b) garantizar que todas las escuelas cuenten con los recursos humanos, servicios públicos, mobiliario y equipo tecnológico necesarios, para evitar el abandono escolar y mejorar la eficiencia terminal, en este sentido, Domínguez et ál. (2013) refieren que la operatividad del programa académico y la formación docente de las y los profesores, son factores asociados al bajo índice de eficiencia terminal, por su parte la United Nations Children's Fund (2001) señala que la privación de una educación de calidad constituye un elemento que presiona la inserción laboral temprana y la consecuente precariedad social.

En otro orden de ideas, se tiene la relación entre delincuencia y educación, la cual es altamente compleja. Existen diferentes tipos de delitos que responden a múltiples factores de propensión a la violencia y la criminalidad, una de estos es la ausencia de oportunidades de educación y empleo (Herrera-Lasso, 2012). Nateras y Zaragoza (2017) señalan que en México la deserción escolar es uno de los factores sociodemográficos determinantes de la delincuencia, los adolescentes que cometen actos delictivos suelen abandonar sus estudios de nivel medio superior. De forma similar, en Uruguay, los adolescentes detenidos suelen ser hombres con un bajo nivel educativo, que tuvieron problemas para su inclusión en las instituciones educativas y el ámbito laboral (Trajtenberg y Eisner, 2015). En alcance a lo anterior, Huang (2016) señala que la relación entre delincuencia y educación tiene su punto de cruce en la deserción durante el bachillerato, pero propone poner especial atención sobre grupos vulnerables desde la educación preescolar, toda vez que asevera que la intervención temprana con niñas y niños en desventaja puede tener efectos positivos de largo alcance, como mejor logro educativo, retención, tasas de empleo, ganancias, prevención del crimen, relaciones familiares y salud. No obstante, los programas deben tener continuidad en los siguientes niveles escolares en temas como el ajuste social, de otra forma su efecto tiende a decaer.

Se deja para el final el análisis de la relación entre delitos y pobreza, que podría ser desde el sentido común lo más fácil de explicar, sin embargo, se requiere una lectura profunda del vínculo entre estas categorías con el fin de evitar la emisión de prejuicios y falacias. Al respecto, autores como Guillén (2006), Ortega (2010), Nateras y Zaragoza (2017), Trajtenberg y Eisner (2015) han declarado que la pobreza no es un factor decisivo para explicar la delincuencia, existen otros factores que activan la predisposición individual a delinquir, así que la pobreza es solo uno de ellos.

La delincuencia y la inseguridad son el resultado de circunstancias y fenómenos complejos; la ruptura del tejido social, la violencia estructural, la impunidad y la corrupción, son tierra fértil para la delincuencia. La población en situación de pobreza no siempre es delincuente ni los ricos son siempre personas honestas. El fenómeno es complejo y juegan un papel relevante otros aspectos como la cohesión comunitaria, los valores predominantes y las oportunidades delictivas (Guillén, 2006). Un dato interesante, aportado por Nateras y Zaragoza (2017), es que las entidades federativas de México con mayor bienestar cuentan con las condiciones necesarias para permitir el establecimiento y desarrollo de grupos delincuenciales, quienes aprovechan factores como la urbanización, la densidad poblacional y las vías de transporte, para maximizar su empoderamiento y margen de operación. En ese orden de ideas, Ortega (2010) señala que los factores socioeconómicos no determinan la comisión de actos delictivos, en contraparte, la impartición de justicia y la eficiencia de los procesos penales sí, con lo que pone el acento sobre el diseño y operación de las instituciones de procuración de justicia y seguridad pública.

\section{EL PROBLEMA DE LA INCLUSIÓN Y LA JUSTICIA SOCIAL}

Después de disertar sobre las generalidades que relacionan entre sí a la educación, pobreza y delincuencia, destaca que el abandono escolar a partir del bachillerato pueda tener relación con la pobreza y la delincuencia, esto lleva a cuestionar si el sistema educativo mexicano está diseñado con un tinte excluyente, que impide la 
inserción plena de miembros de grupos vulnerables en la educación, posteriormente en el mercado laboral $\mathrm{y}$, por tanto, en el sistema social.

$\mathrm{Al}$ respecto, es importante señalar que la inclusión educativa va emparejada con la inclusión social, por lo tanto, se debe explorar la forma en que estas se vinculan. La noción de inclusión educativa ha evolucionado con el tiempo, desde el año 2000, la ONU ha promovido la iniciativa de educación para todos, con lo cual se centra la atención específicamente en la necesidad de llegar a los grupos de estudiantes excluidos y marginados, no solo a los discapacitados (Ainscow, Dyson y Weiner, 2014). De tal forma, la perspectiva de inclusión se enriquece, ahora se contempla la necesidad de brindar atención a aquellas personas que, por cuestiones de identidad sexual, raza, religión, estatus migratorio o alguna otra causa, han sido excluidas del sistema educativo o relegadas al interior de este.

Así, la educación inclusiva puede entenderse como una serie de acciones operadas en la educación formal orientadas a reducir la exclusión social de aquellos colectivos de estudiantes en situación de desventaja (Echetia y Sandoval, 2002). Cabe resaltar que autores como Booth y Ainscow (1998), Echetia y Sandoval (2002), coinciden en que el fin último de la educación inclusiva es equiparar las oportunidades de miembros de grupos vulnerables o marginados para reducir su exclusión en los sistemas educativo y social.

La inclusión o la exclusión no son estados, son un proceso en el que ambos extremos, inclusión-exclusión, están en continua e interminable tensión, de forma que, el avance hacia uno, solo se puede producir por la reducción significativa del otro. Además, este proceso no opera solamente en la escuela, tiene un carácter sistémico, que implica a la comunidad debido a la complejidad de procesos culturales, políticos, económicos y pedagógicos que se encuentran inmersos (Echetia y Sandoval, 2002). Entonces, hablar de inclusión no puede reducirse a un estado ni a lo que ocurre en la escuela, la inclusión en un ámbito no exenta de la exclusión en otros, a esto se suma la relación con actores y grupos de la escuela o externos a esta. No puede haber inclusión educativa si no hay inclusión social y viceversa.

Desde este enfoque de inclusión en la educación, se demanda la existencia de contextos donde subsista una cultura de colaboración que fomente y apoye la resolución de todo tipo de problemas (Ainscow et ál., 2014), esto es, la diversidad sociocultural dentro de las aulas debe enriquecer los patrones de interacción y el proceso de formación integral, antes que convertirse en un obstáculo para la integración social en condiciones de igualdad. Este debe ser el factor que propicie el desarrollo de prácticas que sirvan para todos los alumnos y alumnas, considerando a quienes presenten dificultades especiales como auténticos estímulos del proceso de inclusión (Ainscow, 2001).

De forma tal, las instituciones educativas y la sociedad en general deben enfocarse en promover principios como equidad, solidaridad e igualdad de oportunidades, los cuales son fundamentales para impulsar la inclusión en todos los ámbitos, pero, sobre todo, deben proporcionar los recursos personales, materiales y económicos que garanticen el acceso y permanencia de miembros de grupos vulnerables en el sistema educativo (Calvo y Verdugo, 2012). Por lo tanto, la política educativa debe considerar las diferencias de clase social como uno de los factores a superar, dado que su visibilización permitirá frenar la reproducción de desigualdades sociales mediante el diseño particularizado de oportunidades y condiciones de educación (Cahill, 2015).

Para Arnaiz, Escarbajal y Caballero (2017) es imprescindible crear una cultura escolar en la que todos los estudiantes se sientan valorados, competentes y no excluidos del sistema educativo, el fomento de la vida democrática debe ser una característica esencial en todos los centros escolares. No obstante, de acuerdo con estos autores, se adolece de continuidad de esfuerzos a lo largo de los distintos niveles educativos, en el nivel inicial los docentes poseen niveles de satisfacción más altos con respecto al proceso de inclusión, a diferencia de los docentes de educación primaria. Si esa tendencia siguiera conforme los alumnos avanzan en su educación, al llegar al nivel superior los esfuerzos encaminados a la inclusión serían aún menores y, como se mencionó anteriormente, el impacto sobre la exclusión social de las personas jóvenes sería significativo, pues mientras más avanza el desarrollo psicosocial del individuo se vuelve más importante el papel de la 
escuela como institución facilitadora de la adaptación social y económica, de ahí que Calvo y Verdugo (2012) propongan un proceso inclusivo en todas las etapas educativas que involucre la participación de familiares, docentes, centros escolares y autoridades educativas.

Un elemento a reflexionar es a quiénes y en qué medida se incluye; al respecto, Mascareño y Carvajal (2015) señalan los problemas de inclusión y exclusión que generan las decisiones públicas como, en este caso, la política educativa. Desde la perspectiva de estos autores, así como de Echetia y Sandoval (2002), no puede entenderse la noción de inclusión en términos de una relación dicotómica con la exclusión. En las sociedades modernas hay una relativa individualización de los procesos de inclusión/exclusión debido a la capacidad de las personas para decidir si es conveniente para sus fines integrarse o no en algún nivel de organización social, lo que genera que existan al menos cinco diferentes situaciones de inclusión/exclusión: 1) Autoinclusión/ autoexclusión, las personas deciden por sí mismas participar o no participar. 2) Inclusión por riesgo/exclusión por peligro, las organizaciones toman decisiones que excluyen a ciertos grupos y los expone a peligros, mientras que a otros los incluye en riesgos derivados de dichas decisiones. 3) Inclusión compensatoria, las instituciones implementan programas y acciones para equilibrar situaciones que se asumen temporales, mecanismo tradicional de los Estados de Bienestar. 4) Inclusión en la exclusión, se incluyen grupos o actores en condición de subordinación frente a otros. 5) Subinclusión, se niegan los derechos inherentes a la integración dentro de un sistema social, pero se exige el cumplimiento de obligaciones, también se le podría llamar obediencia a esta situación (Mascareño y Carvajal, 2015).

En el sistema educativo mexicano, en mayor o menor medida, cada una de las situaciones anteriores tiene presencia. La inclusión compensatoria es una práctica común en la política educativa, el otorgamiento de becas a grupos vulnerables (indígenas, migrantes, madres adolescentes, etcétera), los exámenes únicos de acreditación de conocimientos para obtener el grado de licenciatura, así como otro tipo de apoyos otorgados a estudiantes con Necesidades Educativas Especiales.

Se puede decir que también existe inclusión por riesgo/exclusión por peligro, generada por el propio Estado mediante su oferta educativa, la decisión de abrir escuelas en determinada región, de un nivel educativo y un subsistema (Educación Básica, Especial, Técnica y Profesional; Educación General Politécnica y Laboral, Educación para Adultos, etcétera), propicia que los grupos excluidos se mantengan al margen de los demás sectores sociales y que las oportunidades de desarrollo sigan estando lejos de ellos; por ejemplo, para la población general tiene un valor distinto asistir a un bachillerato general frente al bachillerato tecnológico, de igual forma, una secundaria técnica o telesecundaria se valúa menos en comparación con una secundaria regular, sin embargo, en zonas marginadas se sigue promoviendo la apertura de bachilleratos tecnológicos y secundarias técnicas.

La inclusión en la exclusión puede ser apreciada en la creación de universidades interculturales diseñadas para la población indígena, las cuales parecieran ser una señal de integración parcial, como si el mensaje fuera: los indígenas deben estudiar, pero en sus propias escuelas, sin mezclarse con el resto de la población.

En cuanto a la subinclusión, se puede presentar como ejemplo a los estudiantes con Necesidades Educativas Especiales que son inscritos en escuelas regulares, bajo el argumento de equidad, sin embargo, el resultado final suele ser que, debido al rechazo de docentes, padres de familia y compañeros, estos permanezcan en la institución en condiciones de aislamiento o atención precaria, pese a que se les exige el cumplimiento de los mismos deberes del resto de la comunidad escolar.

No menos importante es la cantidad de personas que decide no continuar sus estudios, por diversas razones hay quienes se autoexcluyen del sistema educativo: porque no les gusta estudiar, prefieren formar una familia, eligen trabajar o aprender un oficio, etcétera. De tal forma, sería prudente repensar el objetivo de inclusión en el sentido casi romántico que se plantea desde la política educativa: educación para todos. Efectivamente, para todos debe asegurarse el derecho a la educación, mas no puede educarse a todos por igual.

Para comprender objetivamente las implicaciones y el origen de las condiciones de inclusión/exclusión que caracterizan a México, más allá de ideologías y aspiraciones, se recurre a los postulados de Georg Simmel 
sobre la diferenciación social e individualización, mediante los cuales puede ilustrarse que la desigualdad es ineludible, pero esta no es sinónimo de exclusión.

Simmel (2017) es uno de los pioneros en el estudio de la individualidad y la fragmentación social, podría decirse que su sociología se refiere a la interacción, por lo que centra sus discusiones en los conflictos y contradicciones sociales. Si bien no hace un análisis directo sobre las variables discutidas en este documento pobreza y delincuencia-, el dinero ocupa un lugar importante en su obra, como determinante del intercambio social, de ahí que sus postulados sirvan como base para analizar la pobreza, las condiciones socioeconómicas que determinan la delincuencia y el papel de la educación en torno a la fragmentación social.

Con la modernidad, la sociedad deja de ser una unidad absoluta y cerrada, la relativización y fragmentación del mundo conlleva la diferenciación social, entendiéndola como división del trabajo, especialización y, sobre todo, pérdida de la dependencia del individuo respecto del grupo. Por lo tanto, la diferenciación tiene como efecto el surgimiento de la individualidad y la libertad modernas. La independencia tendencial de los grupos de pertenencia, desarrolla lo que es propio y único de cada individuo. A pesar del número de círculos sociales en que participa el individuo, este ejerce su libertad de acción y se diferencia (Simmel, 2017). Las formas de socialización (vergesellschaftung), entonces, operan entre seres de diferencias, que generan formas de intercambio de efectos (wechselwirkungen) o, dicho de otra forma, relaciones de acción recíproca. Con esto, se enfatiza el desarrollo de la individualidad, pues existe independencia de las formas sociales respecto de los individuos particulares (Rammstedt y Cantó i Milá, 2007).

En las sociedades modernas, el dinero es uno de los principales medios por el que se encuentran interconectados todos y a la vez se constituye en un factor de diferenciación, grupal e individual. En suma, con la modernidad surge el individuo, mediante un proceso de diferenciación que implica la existencia de desigualdad económica.

Las desigualdades al interior de una sociedad no deberían debilitar ni resquebrajar. La modernidad configura una relación entre lo individual y lo colectivo, por medio de un proceso de moralización que dibuja la forma en que deben operar los procesos de individualización y diferenciación, así se construyen las líneas rectoras de las sociedades modernas. Las partes del todo se imbrican, lo cual induce la cohesión y el crecimiento del todo, esto lo fortalece y posibilita el desarrollo de una cultura y formas de asociación interna. En tanto los procesos se institucionalicen, como estado de derecho y moral, el todo será más independiente frente a las partes y las partes tendrán que entregar cada vez menos al todo. Pero para alcanzar dicho estado es necesario que las exigencias del grupo social al individuo estén diferenciadas de manera tajante. La sociedad espera que haya alta cohesión del individuo con el grupo para que este acepte la diferenciación (división de trabajo) que le resulta conveniente, sin embargo, al mismo tiempo el individuo busca diferenciarse del grupo para ser único y libre. En esta tensión generada entre cohesión y libertad, el punto de equilibrio estará dado por la cultura, la cual configura tareas individuales y grupales de forma que se diferencien en el mismo grado, así el individuo entiende que hay circunstancias momentáneas que exigen cohesión grupal para cumplir las tareas que le corresponden y que, al mismo tiempo, abren la posibilidad de ejecutar otras que lo diferencian (Simmel, 2017). En términos económicos, esto podría traducirse en reducir la brecha entre pobres y ricos. Siempre habrá sociedades en las cuales sus miembros se distingan por estratos sociales, sin embargo, el grado de desigualdad económica debe ser tal que permita encontrar un punto de equilibrio que, al igual que la cultura, favorezca la cohesión social.

Pareciera entonces que la diferenciación, al requerir tareas particulares para cada actor y ubicarlo en una posición distinta dentro del sistema social, obliga a que exista desigualdad, no obstante, esa sería una interpretación errónea, la modernidad persigue otra meta: una diferenciación social dada por la desigualdad de esfuerzos personales desplegados, de modo que el progreso estará activado por la igualdad de oportunidades y será el individuo quien determine su propio progreso, no la provisión comunitaria. En sociedades en las que aún operan lógicas premodernas, la diferenciación sí puede derivar en desigualdad, debido a que las instituciones sociales y los patrones culturales tienden a preservar prácticas que perpetúan 
la heterogeneidad social y la inmovilidad social, es decir, se busca la igualdad independientemente del grado de esfuerzo, simplemente por ser miembro de un grupo se espera obtener iguales recompensas, además de reproducir prácticas sociales que perpetúan el statu quo y evita que los grupos de poder dejen de estar al frente de la sociedad. Al estar en una sociedad donde la riqueza no se obtiene por méritos individuales sino por el aprovechamiento de recursos socioculturales informales, el punto de equilibrio se rompe y se perciben injusticias. Las formas de estar con otros, pueden ser de dominación, cooperación, conflicto, confianza, etcétera, y en todas hay intercambio de efectos. Las experiencias cotidianas conectan los sentidos y las emociones, el cuerpo con la sociedad, por medio de interacciones jerarquizadas se puede sentir desprecio hacia otro que es considerado inferior (Sabido, 2007). Así, ser diferente no corresponde a la división del trabajo o a los méritos individuales, responde a la pertenencia a grupos que históricamente han sido relegados o privilegiados.

Lo anterior lleva a otro debate, si en una sociedad con rasgos premodernos la diferenciación conlleva el riesgo de exclusión a los grupos menos favorecidos ¿cómo se vinculan la diferenciación y la justicia social?, ¿qué papel juega la educación en torno a la justicia social? Para estos fines se recupera a dos teóricos emblemáticos: John Rawls y Amartya Sen. El objeto primario de la justicia, para Rawls (1995), es la estructura básica de la sociedad, este es el modo en que las instituciones sociales fundamentales distribuyen los derechos y deberes elementales, lo que determina la división de las ventajas de la cooperación social.

El concepto de justicia de Rawls (1995) está construido en relación con la idea de posición original, la cual es una situación imaginaria de igualdad en donde la justicia será producida sin que el propio actor lo determine. Esto definirá un aparato social básico para una sociedad realmente justa. Bajo este principio, se busca la igualdad de distribución sobre la base de una institución que garantice las mismas libertades y oportunidades generales (Huang, 2016).

Por su parte, Sen (2009) propone quitar del centro de atención la naturaleza de las instituciones, puesto que la justicia guarda mayor relación con la forma en que las personas viven sus vidas. Sen reconoce la aportación de Rawls en sus planteamientos sobre la justicia, el cual asume que hay una distribución natural de bienes, por lo que aquellas personas con el mismo nivel de talento y habilidades, asumiendo que tienen el mismo interés por usarlos, deberían tener la misma prospectiva de éxito, independientemente de su posición inicial en el sistema social (Huang, 2016). Sin embargo, Sen (2009) propone el concepto de igualdad de oportunidades como principio de justicia. Así, pese a la naturaleza de las instituciones, la distribución natural de bienes o la identificación de esquemas sociales ideales, la justa equidad de oportunidades es la prioridad, lo que dirigiría el diseño de políticas públicas hacia la implementación de acciones las cuales aseguren que el remanente de desigualdades está a favor de los menos aventajados.

De acuerdo con Huang (2016), la teoría liberal de Rawls fue la base para la igualdad de oportunidades educativas. Mas, la teoría de justicia de Sen ha promovido cambios; este último enfatiza la libertad de escoger entre diferentes clases de vidas y la responsabilidad inherente ante lo que hacemos, lo que llevó a cambiar la idea de igualdad en los sistemas educativos, desde la que se plantea que todos deben gozar de las mismas condiciones de educación, a la de equidad de oportunidades con un trato diferenciado que en cierta forma compense las condiciones de desventaja que afrontan algunos actores. Desde la perspectiva de Sen, el concepto de oportunidades de educación asigna más libertad al individuo. La atención sobre el enfoque de capacidad, no es solo en torno a lo que la persona hace, sino lo que es capaz de hacer si elige hacer uso de esa oportunidad (Huang, 2016).

De tal manera que, en un escenario ideal en el que existen instituciones sociales consolidadas, la educación es uno de los principales medios de movilidad social, que promueve la diferenciación social al mismo tiempo que promueve la justicia social al brindar oportunidades equitativas para el desarrollo individual. Sin embargo, en una sociedad donde las instituciones operan mediante prácticas premodernas, la educación es un bien inaccesible para los sectores más vulnerables de la población, sobre todo en el nivel superior, generando 
que no se pueda hablar de diferenciación social sino de mecanismos de exclusión y esto, a su vez, definirá condiciones de injusticia social.

De acuerdo con Simmel (2017), en la premodernidad la tendencia era castigar al círculo social por lo que hacía uno de sus miembros, en el caso del sistema educativo mexicano aún se puede apreciar esa tendencia hacia los grupos vulnerables. Ser indígena, pobre o habitante de una población marginada, estigmatiza y obstruye el ingreso a la educación media superior y superior, sin importar sus capacidades individuales ni el interés por usarlas. Los programas que pretenden apoyar a los grupos vulnerables son un simple paliativo económico que no abre oportunidades plenas para culminar la educación superior y, por consecuencia, tampoco para insertarse en el mercado laboral.

Como consecuencia, en sociedades premodernas, la fragmentación social es mayor, tal es el caso de las entidades federativas que operan sistemas educativos sin procesos institucionalizados, en donde los grupos fácticos de poder controlan la educación, como Chiapas, Oaxaca y Guerrero. Esto repercute en un menor promedio de escolaridad y a su vez presentan mayores valores en pobreza (ver tabla 1). En estos estados, los actores periféricos quedan excluidos del sistema educativo o su inclusión es desde la exclusión o la subinclusión. La pobreza va más allá de las carencias materiales y contribuye a la exclusión social, las niñas y niños tienden a reducir su motivación al aprendizaje, retrasar su desarrollo cognitivo, tener un desempeño bajo, menor participación en actividades extra curriculares, bajas aspiraciones de educación superior, interrumpen su trayectoria académica, incrementan el riesgo de analfabetismo e incrementan la posibilidad de abandono escolar (Canadian Teachers' Federation, 2009).

Por lo tanto, no solo debe trabajarse en mejorar la calidad de educación que reciben las niñas y los niños en situación de pobreza; es imperativo incluir políticas sociales y económicas operadas en la escuela que posibiliten ampliar su impacto (Canadian Teachers' Federation, 2009), lo cual permitiría atender la prevención del delito, entre otras cosas, al evitar el abandono escolar y la consecuente imposibilidad de acceso a fuentes de trabajo. De esta forma, se rompería con la lectura simplista de la pobreza como causa de la delincuencia, para dar paso a la consideración de factores socioeconómicos, de desempeño institucional y de educación.

\section{Conclusiones}

Podría decirse que uno de los mayores fracasos del sistema educativo mexicano es el abandono escolar en el nivel medio superior, lo cual a su vez parece estar relacionado con la participación de las personas jóvenes en actos criminales y con la imposibilidad de acceder al mercado laboral o, en el caso de quienes lo logran, con una incorporación laboral precaria. En ese sentido, la falta de oportunidades para continuar un proceso formativo que culmine en la educación superior, preserva los círculos de pobreza en las familias marginadas y vulnera socialmente a dichos actores, sumando un factor más para que estos participen en actos delictivos.

Las entidades federativas con mayor pobreza son aquellas que también presentan menores promedios de escolaridad y, aunque la relación con el número de delitos del fuero común no es tan clara, se puede afirmar que la pobreza es un factor que incentiva la comisión de delitos, como afirman Guillén (2006), Ortega (2010), Nateras y Zaragoza (2017), Trajtenberg y Eisner (2015). La Comisión Económica para América Latina y el Caribe (2007) declara que la educación constituye el mecanismo privilegiado para la superación de la pobreza, los mayores logros educativos tienen mayores retornos intergeneracionales y una mayor educación permite una mayor movilidad socio-ocupacional ascendente. De acuerdo con este organismo, a mayor nivel de educación formal, menor es la probabilidad de ser pobre o caer en la pobreza. En este sentido, cobra relevancia la necesidad de implementar acciones encaminadas a la inclusión educativa y social de las y los jóvenes, no solo con medidas paliativas que se enfoquen en el acceso, entendido como aumentar la cobertura educativa, sino también en la mejora de la calidad de los servicios educativos para garantizar que las y los jóvenes terminen sus estudios, sobre todo en las zonas históricamente rezagadas. 
El espíritu de las acciones diseñadas e implementadas con el fin de mejorar la inclusión educativa, debe tener un carácter profundamente social, desde dos acepciones: incorporar en el proceso no solo a los actores educativos, también a las familias y las comunidades en general; promover la inserción y participación igualitaria de todos los actores no solo en el sistema educativo, también en el social y el laboral. Las acciones de inclusión educativa se podrán traducir en resultados de justicia social, en la medida que estas amplíen su alcance, más allá de lo escolar, e incorporen principios y prácticas que respondan a la modernidad. No se puede aspirar a ser una sociedad con mayor bienestar y estabilidad social si las instituciones operan bajo prácticas premodernas las cuales excluyen a los grupos que siempre han sido relegados. En las condiciones actuales, el sistema educativo mexicano plantea inequidad en las oportunidades de desarrollo de capacidades y no promueve la justicia social. El éxito educativo no depende del talento ni el esfuerzo individual.

Es fundamental dejar de entender la inclusión como algo contrapuesto a la exclusión, pero sobre todo como algo que persigue la heterogeneidad social. En el modelo educativo mexicano debería considerarse que la educación debe ser diferenciada, no puede educarse de la misma forma a todos, pese a que esto se contraponga a los principios políticamente correctos de inclusión. Esta afirmación puede parecer arriesgada, sin embargo, de acuerdo con autores como Ainscow (2001), Nateras y Zaragoza (2017), Trajtenberg y Eisner (2015) y Huang (2016), está sustentada en la idea de formar con especial atención y con fines particulares a las niñas y niños provenientes de grupos vulnerables que se distinguen por alto riesgo de fracaso escolar y la consecuente participación en actos delictivos. Para tener éxito en la inclusión educativa, no es suficiente ampliar la cobertura, la intervención debe ser acorde a las necesidades de cada grupo, solo así se podrá incidir en la prevención de la delincuencia y el combate a la pobreza.

Como se señala en las líneas anteriores, una de las vías para abatir la pobreza y la delincuencia es la educación, pero no la educación que actualmente se imparte, primero tendrían que resolverse los problemas de calidad académica, la deserción en nivel medio superior y superior, así como la cobertura en estos niveles educativos. Además de los vicios que arrastra el sistema educativo en cuanto a los mecanismos de ingreso, permanencia y promoción en el servicio, formación continua y salarios de docentes.

\section{REFERENCIAS}

Ainscow, M. (2001). Desarrollo de escuelas inclusivas: ideas, propuestas y experiencias para mejorar las instituciones escolares. Madrid: Narcea

Ainscow, M., Dyson, A. y Weiner, S. (2014). De la exclusión a la inclusión. Una revisión literaria internacional en camino para responder a los estudiantes con necesidades educativas en las escuelas, En-clave Pedagógica, 13, 13-30

Arnaiz, P., Escarbajal, A. y Caballero, C. M. (2017). El impacto del contexto escolar en la inclusión educativa. Revista Nacional e Internacional de Educación Inclusiva, 10 (2), 195-210

Booth, T. y Ainscow, M. (1998). From them to us. An international study of inclusion in education. Londres: Routledge

Cahill, K. (2015). Seeing the wood from tha trees: a critical policy analysis of intersections between social class inequality and education in twenty-first century Ireland. International Electronic Journal of Elementary Education, 8 (2), 301-316.

Calvo, M. I. y Verdugo, M. A. (2012). Educación inclusiva, ¿una realidad o un ideal? EDETANIA, 41, 17-30

Canadian Teachers' Federation (2009). Supporting Education... Building Canada. Child poverty and schools. Recuperado de http://www.ctf-fce.ca/Research-Library/FINAL_Hilldayleavebehind_eng.pdf

Comisión Económica para América Latina y el Caribe (2007). Cohesión social: inclusión y sentido de petenencia en América Latina y el Caribe. Santiago de Chile: Naciones Unidas

Comisión Económica para América Latina y el Caribe (2009). Impacto social y económico del analfabetismo: modelo de análisis y estudio piloto. Santiago de Chile: Naciones Unidas

Consejo Nacional de Evaluación de la Política de Desarrollo Social (2016). Medición de la pobreza a nivel nacional, 2016. Recuperado de https://www.coneval.org.mx/Medicion/MP/Paginas/Pobreza_2016.aspx 
Di Lorenzo, L. N. y Rosales, G. A. (2013). Analfabetismo y pobreza en San Luis: un estudio descriptivo y comparativo, Fundamentos en Humanidades, 14 (28), 29-49

Domínguez, D., Sandoval, M. C., Cruz, F. y Pulido, A. R. (2013). Problemas relacionados con la eficiencia terminal desde la perspectiva de estudiantes universitarios. Revista Iberoamericana sobre Calidad, Eficacia y Cambio en Educación, 12 (1), 25-34

Echetia, G. y Sandoval, M. (2002). Educación inclusiva o educación sin exclusiones. Revista de Educación, 327, 31-48

Guillén, F. (2006). Las políticas locales de prevención y seguridad en Europa. Revista Catalana de Seguretat Pública. $16,59-79$

Herrera-Lasso, L. (2012). Factores que propician la violencia y la inseguridad: apuntes para una estrategia integral de seguridad pública en México. México: Grupo Coppan SC Manuscript

Huang, B. R. (2016). Social justice and capacity for Self-development in Educational Systems in European Union. Education Provision to Every One: Comparing Perspectives from Around the World. BCES Conference Books, 14(1)

Li, J. (2016). Who decided college access in chinese secondary education? Rural-urban inequality of basic education in contemporary China. Universal Journal of Educational Research, 4 (10), 2392-2405

Mascareño, A. y Carvajal, F. (2015). Los distintos rostros de la inclusión y la exclusión. Revista CEPAL, 116, 131-146

Nateras, M. y Zaragoza, D. (2017). La pobreza como indicador de generación de la violencia y la delincuencia en México. En F. Betancourt (Coord.). Reflexiones sobre el estado de derecho, la seguridad pública y el desarrollo de México y América Latina (pp. 221-250). México: UNAM

Ortega, J. A. (2010).¿Pobreza igual a delito? Los factores socio-económicos del crimen y el derecho humano a la seguridad pública. México: Comisión de Derechos Humanos del Estado de México

Rammstedt, O. y Cantó i Milá, N. (2007). Georg Simmel (1858-1918). En O. Sabido (Coord.). Georg Simmel. Una revisión contemporánea. Anthropos: Barcelona

Rawls, J. (1995). Teoría de la justicia. México: Fondo de Cultura Económica

Sabido, O. (2007). El sentir de los sentidos y las emociones en la sociología de Georg Simmel. En O. Sabido (Coord.). Georg Simmel. Una revisión contemporánea. Anthropos: Barcelona

Secretaría de Educación Pública (2017). Nuevo Modelo Educativo. México: SEP

Secretariado Ejecutivo del Sistema Nacional de Seguridad Pública (2017). Incidencia delictiva del fuero común 2017. Recuperado de https://bit.ly/2IraSmZ

Sen, A. (2009). La idea de justicia. México: Santillana Ediciones

Simmel, G. (2017). Sobre la diferencia social. Investigaciones sociológicas y psicológicas. Barcelona: Gedisa Editorial

Sistema Nacional de Información y Estadística Educativa (2017). Estadística e Indicadores Educativos por Entidad Federativa. Recuperado de http://www.snie.sep.gob.mx/x_entidad_federativa.html

Trajtenberg, N. y Eisner, M. (2015). Towards a more effective violence prevention policy in Uruguay. Montevideo: University of Cambridge, Universidad de la República, Administración Nacional de Educación Pública

United Nations Children's Fund (2001). Educación, pobreza y deserción escolar. Santiago de Chile: UNICEF

CC BY-NC-ND 